\title{
CONTROVERSIAS PIAGET-VYGOTSKI EN PSICOLOGÍA DEL DESARROLLO
}

\author{
Rosalía Montealegre* \\ Universidad Nacional de Colombia - Bogotá, D.C., Colombia
}

\author{
Recibido, marzo 17/2014 \\ Concepto evaluación, agosto 28/2014 \\ Aceptado, diciembre 13/2014
}

\begin{abstract}
Referencia: Montealegre, R. (2016). Controversias Piaget-Vygotski en Psicología del Desarrollo. Acta Colombiana de Psicología, 19(1), 271-283. DOI: 10.14718/ACP.2016.19.1.12
\end{abstract}

\section{Resumen}

\begin{abstract}
En este artículo de reflexión se analizan tres controversias originadas a partir de la psicología lógica operatoria de Piaget y de la psicología histórico-cultural de Vygotski, sobre: el lenguaje egocéntrico y el lenguaje para sí en el niño preescolar; el simbolismo y la situación imaginaria en el juego infantil; el desarrollo cognitivo real y el desarrollo cognitivo potencial. Se analizan las controversias a partir de "la unidad de análisis": la lógica de la acción o de la operación, y la equilibración, en Piaget; y la acción mediada por instrumentos materiales, semióticos y humanos, en Vygotski. El trabajo se orienta hacia la posición de Vygotski y presenta investigaciones neo-vygotskianas relacionadas con: 1. El lenguaje egocéntrico como instrumento planificador y regulador de la acción intelectual, en el preescolar. 2. El juego infantil, en el cual el niño aprende a definir su conducta por el sentido de la situación imaginaria creada. 3. El desarrollo cognitivo potencial define el aprendizaje; y el aprendizaje organizado es fuente de desarrollo. Los objetivos del trabajo van orientados a: 1. Analizar las diferencias conceptuales y metodológicas entre la psicología de Piaget y la de Vygotski. 2. Precisar la investigación neo-vygotskiana en psicología del desarrollo.

Palabras clave: Piaget-Vygotski, neo-vigotskianos, lenguaje egocéntrico, juego infantil, desarrollo cognitivo.
\end{abstract}

\section{PIAGET-VYGOTSKY CONTROVERSIES IN DEVELOPMENTAL PSYCHOLOGY}

\begin{abstract}
This review article analyzes three controversies originating from the Piaget's logical operational psychology and Vygotsky's cultural-historical psychology on: the egocentric speech and the self-talk in the pre-school child; the symbolism and imaginary situations during children's play; the real cognitive development and the potential cognitive development. The controversies are analyzed from the "unit of analysis": the logic of the action or the operation, and equilibration, in Piaget; and the action mediated by material semiotic and human instruments, in Vygotski. This paper leans towards Vygotsky's position and presents neo-Vygotskian research related to 1 . The egocentric speech as a planning and regulating instrument of intellectual action in the preschooler. 2. Children`s play where the youngsters learn to define their behavior with the created imaginary situation. 3 . The potential cognitive development defines learning; and the organized learning is a source of development. The objectives of this research are oriented to: 1 . Analyze the conceptual methodological differences between the psychology of Piaget and that of Vygotski. 2. Clearly state the neo-Vygotskian research in developmental psychology.

Key words: Piaget-Vygotski, neo-Vygotskians, egocentric speech, children's play, cognitive development.
\end{abstract}

\footnotetext{
* Rosalía Montealegre, Ph.D en Psicología, Universidad M.V. Lomonósov de Moscú (Rusia). Psicóloga, Pontificia Universidad Javeriana (Bogotá, Colombia). Docente Pensionada, Universidad Nacional de Colombia. Tels. Bogotá, Colombia (57 1) 2110709; (57) 3108812307. rosaliamh@gmail.com Líneas de investigación: Psicología del Desarrollo y Psicología Cultural Educativa a partir de la "psicología histórico-cultural" de la escuela vygotskiana.
} 


\title{
CONTROVÉRSIAS PIAGET-VYGOTSKI EM PSICOLOGIA DO DESENVOLVIMENTO
}

\author{
Resumo
}

\begin{abstract}
Neste artigo de reflexão, analisam-se três controvérsias originadas a partir da psicologia lógica operatória de Piaget e da psicologia histórico-cultural de Vygotski sobre: a linguagem egocêntrica e a linguagem para si na criança pré-escolar; o simbolismo e a situação imaginária no jogo infantil; o desenvolvimento cognitivo real e o desenvolvimento cognitivo potencial. Analisam-se as controvérsias a partir da "unidade de análise": a lógica da ação ou da operação, e do equilibração em Piaget; e a ação mediada por instrumentos materiais, semióticos e humanos, em Vygotski. Este trabalho está orientado ao posicionamento de Vygotski e apresenta pesquisas neovygotskianas relacionadas com: 1) a linguagem egocêntrica como instrumento planejador e regulador da ação intelectual na criança pré-escolar; 2) o jogo infantil, em que a criança aprende a definir seu comportamento pelo sentido da situação imaginária criada; 3) o desenvolvimento cognitivo potencial define a aprendizagem; a aprendizagem organizada é fonte de desenvolvimento. Os objetivos deste trabalho vão orientados a: analisar as diferenças conceituais e metodológicas entre a psicologia de Piaget e a de Vygotski; precisar a pesquisa neovygotskiana em psicologia do desenvolvimento.

Palavras-chave: Piaget-Vygotski, neovigotskianos, linguagem egocêntrica, jogo infantil, desenvolvimento cognitivo.
\end{abstract}

\section{INTRODUCCIÓN}

En 2016 se cumplen 120 años del nacimiento de Jean Piaget (1896-1980) y de Lev Semiónovich Vygotski (18961934). Aunque estos dos teóricos e investigadores de la psicología comenzaron su producción científica en la misma época, no intercambiaron ideas ni debatieron entre sí sus trabajos debido a circunstancias políticas que rodearon la vida y la obra de Vygotski. Sin embargo, sus postulados teóricos en psicología del desarrollo están entrelazados y han suscitado una serie de debates respecto al lenguaje, al juego infantil y al desarrollo cognitivo. Vygotski, en su libro "Pensamiento y Lenguaje", publicado en 1934 (Vygotski, 1934/1993) comenta y critica la obra de Piaget de 1923 y 1924 (Piaget, 1923/1983; 1924/1973) sobre el egocentrismo referido a la inhabilidad inicial del niño para descentrarse. Piaget (1959/1985) escribe el texto Commentaires sur les remarques critiques de Vygotski, veinticinco años más tarde, al conocer las críticas de Vygotski en "Pensamiento y Lenguaje", donde concreta algunos momentos de su comprensión del lenguaje egocéntrico. Trata de ver si se justifican las observaciones críticas de Vygotski a la luz de sus últimos trabajos. Así, Piaget precisa que por "egocentrismo" entiende "la incapacidad inicial del niño de descentrarse, de cambiar la perspectiva cognoscitiva dada", lo que no está ligado en ninguna manera con la hipertrofia de la conciencia del propio "yo". El egocentrismo cede lugar a la descentración o proceso de superación de la posición mental propia.

Al analizar el desarrollo psicológico del ser humano en la psicología lógica operatoria de Piaget y en la psicología histórico-cultural de Vygotski, se hace necesario determinar en estas concepciones teóricas las unidades de análisis que guían sus aspectos metodológicos e investigativos.
Las unidades de análisis en la psicología lógica operatoria de J. Piaget son: la lógica de la acción o de la operación; y la equilibración

Para Piaget (1956/1967; 1972/1980; 1964/1981) el desarrollo cognitivo está relacionado con la lógica de las acciones, la ejecución y coordinación de dichas acciones; con la lógica de las operaciones concretas y formales, y con la lógica de las significaciones.

Piaget e Inhelder (1969/2007), en el libro Psicología del niño, consideran que el desarrollo mental es una sucesión de tres grandes construcciones: a) la construcción de los esquemas de acción sensorio-motores; b) la construcción de las relaciones semióticas, que reconstruye los esquemas de acción anteriores en un nuevo plano de la representación, y constituye el conjunto de las operaciones concretas y las estructuras de cooperación; y finalmente, c) la construcción del pensamiento formal, que reestructura las operaciones concretas subordinándolas a nuevas estructuras; esta construcción tiene lugar durante la adolescencia y continúa toda la vida.

Esta división del desarrollo en grandes períodos o estadios, obedece a los siguientes criterios: a) a un orden de sucesión constante, aunque los promedios de edades pueden variar según el grado de inteligencia y el ambiente social; b) a una estructura de conjunto que caracteriza cada estadio; y c) a la integración de las estructuras de conjunto que no se sustituyen unas a otras: cada estructura resulta de la anterior y prepara la siguiente.

Por otra parte, Piaget (1972/1980; 1964/1981; 1975/2012) analiza en el ser humano el proceso de equilibración de sus estructuras cognitivas. Considera la equilibración como el problema central del desarrollo, y plantea el "modelo de equilibrio por compensación" entre las perturbaciones exteriores y las actividades del sujeto. En el ser humano, 
las perturbaciones exteriores son un obstáculo para el logro de un objetivo, para los procesos de asimilación o incorporación de un elemento exterior (objeto, acontecimiento, etc.) en un esquema sensorio-motor o conceptual. Al recurrir a la noción de equilibración, en el análisis del desarrollo cognitivo, explica la génesis de las estructuras mentales y el paso de las regulaciones preoperatorias a las operatorias propiamente dichas.

Para Piaget (1975/2012), en el ser humano la equilibración progresiva de las estructuras cognitivas es un proceso cuyas manifestaciones se modifican en cada estadio del desarrollo cognitivo del individuo, en el sentido de un mejor equilibrio, tanto en su estructura cualitativa como en su campo de aplicación.

La unidad de análisis en la psicología histórico-cultural de L.S. Vygotski: la acción mediada por instrumentos (mediación instrumental)

Vygotski, al desarrollar su enfoque histórico-cultural en psicología (1924-1934), aplica la mediación instrumental al estudio de los procesos cognitivos y al análisis sociocultural. Un instrumento material, un signo (instrumento semiótico o psicológico), y un ser humano, llevan a una nueva composición del comportamiento y a una reconstrucción de la estructura del proceso psíquico. En la solución de una tarea, el procedimiento está orientado por el instrumento, y los procesos psíquicos proceden según este, formándose determinados actos instrumentales que llevan al desarrollo de las Funciones Psíquicas Superiores (FPS).

Vygotski(1931/1983;1930/1984; 1930/1991) considera necesario que el ser humano, para organizar, dominar y reconstruir una operación psíquica o cualquier función psíquica superior (pensamiento productivo y discursivo, la memoria lógica, el lenguaje significativo, etc.), debe introducir signos o instrumentos culturales (signos lingüísticos; signos nemotécnicos; instrumentos materiales y psicológicos; sistemas de escritura y numeración, de esquemas y diagramas, etc.) que sirvan de medio auxiliar en la solución de cualquier tarea de asimilar, recordar, reconocer, comunicar, comparar, elegir, evaluar y ponderar algo.

Vygotski (1931/1983; 1934/1993), al estudiar el desarrollo ontogenético, plantea la ley genética del desarrollo cultural: toda función psíquica aparece en escena dos veces en dos planos, primero social y luego psicológico; primero, entre las personas de manera interpsíquica, y luego en el interior del sujeto, de modo intrapsíquico. Bajo esta ley psicogenética, para desarrollar diferentes tipos de acciones (intelectuales, comunicativas, etc.) es indispensable lograr primero el dominio interpsíquico (interacción con otro), y posteriormente, el intrapsíquico (manejo interior de las acciones). La acción exterior se vuelve representación men- tal, en donde el signo y su empleo han sido determinantes. El signo es inicialmente un medio de relación social, de influencia sobre otras personas, y después, un medio para influir sobre sí mismo. El signo mediatiza la relación del ser humano con el otro y consigo mismo. Los signos se interponen entre cualquier función natural psíquica del ser humano y su objeto, cambiando de raíz las propiedades de dicha función.

En concreto, en la psicología histórico-cultural, la unidad de análisis es la acción mediatizada por instrumentos: materiales, semióticos (signos) y humanos (un adulto o par significativo) que lleva a ampliar el comportamiento del sujeto en sus interacciones con el médio, con los otros y consigo mismo (Zínchenko, 1993; Wertsch, 1985/1988).

Teniendo en cuenta lo anterior, los objetivos de este artículo son los siguientes: 1. Analizar las diferencias conceptuales y metodológicas entre la psicología lógica operatoria de J. Piaget y la psicología histórico-cultural de L.S. Vygotski. 2. Precisar, a partir de la conceptualización teórica y de la unidad de análisis de la psicología vygotskiana, el curso de la acción investigativa en el estudio del lenguaje, el juego y el desarrollo cognitivo.

\section{CONTROVERSIAS PIAGET-VYGOTSKI}

El conocido "Debate Vygotski-Piaget" sobre el fenómeno del "lenguaje egocéntrico" (Piaget, 1923/1983; 1924/1973) y del "lenguaje para sí" (Vygotski, 1934/1993), a comienzos de la década de los 30 , se presenta en primer lugar. En segundo lugar, se aborda la "Controversia sobre el Juego Infantil" presentando la posición de Piaget sobre el simbolismo en el juego; y la de Vygotski, acerca de la situación imaginaria. En tercer lugar, la "Controversia sobre el Desarrollo Cognitivo" precisa la posición de Piaget: "el nivel real de desarrollo cognitivo determina el aprendizaje"; y la de Vygotski: "el desarrollo cognitivo potencial define el curso del aprendizaje".

Debate Vygotski-Piaget sobre el lenguaje egocéntrico en el niño

Piaget (1923/1983; 1924/1973) considera el lenguaje egocéntrico como uno de los síntomas del pensamiento egocéntrico del niño de edad preescolar: al hablar, el niño no trata de entender el punto de vista de quien lo escucha. El pequeño ve la realidad a través de su propia óptica, sin tomar conciencia de ello. Su propia visión aparece como absoluta. Piaget concluye que el niño es un "prisionero de su posición mental". Opone el lenguaje egocéntrico al lenguaje socializado. Considera que el lenguaje egocéntrico es genéticamente primario con respecto al socializado. 
Para Piaget, el lenguaje egocéntrico o "lenguaje desde el propio punto de vista" surge a causa de la insuficiente socialización del lenguaje, inicialmente individual. A medida que el niño crece, el lenguaje egocéntrico disminuye, siendo sustituido por el lenguaje socializado y, al final, desaparece. El lenguaje en el niño se hace socializado y comunicativo sólo hacia los siete u ocho años de edad, al surgir una acción lógica operatoria (Montealegre, 1990; 1991/1994; 1998).

Piaget (1932/1977) afirma que la consecuencia principal del egocentrismo infantil es el sincretismo. El niño descuida las vinculaciones objetivas en favor de las propias subjetivas. El sincretismo penetra todo el pensamiento del niño, dificulta el análisis objetivo de los fenómenos y los razonamientos deductivos.

Para Piaget (1936/1969), otra consecuencia del egocentrismo es la insensibilidad del niño a las contradicciones; por ejemplo, puede afirmar inicialmente que los barcos de madera navegan porque son ligeros, y unos minutos después, argumentar que los barcos grandes no se hunden porque son pesados. La insensibilidad a las contradicciones está provocada porque el niño en edad preescolar razona con transducciones (transposiciones), al moverse su pensamiento de una particularidad a otra, sin necesidad lógica, sin apoyarse en definiciones exactas.

En síntesis, la teoría piagetiana señala las raíces del egocentrismo intelectual en el carácter asocial del niño y en el carácter peculiar de su actividad práctica. El egocentrismo del pensamiento infantil en esta teoría es considerado el resultado de desfigurar las formas sociales de pensamiento.

Algunos autores han precisado la polémica a partir del nombre que le han dado a este fenómeno; al respecto, Flavell y otros (1968), Kohlberg y otros (1968), proponen el término private speech para designar las expresiones del niño no dirigidas y no adaptadas al escucha. En el Debate Vygotski-Piaget se ha investigado la dependencia del private speech con respecto a la edad, el sexo, la nacionalidad, el cociente intelectual, la dificultad de la tarea, y el papel regulador de los adultos en la solución de las tareas del niño.

A diferencia de Piaget, Vygotski (1934/1993) formula la hipótesis del carácter inicialmente social del lenguaje $y$ del surgimiento del lenguaje egocéntrico como resultado de la insuficiente individualización del lenguaje. En el niño, su primario lenguaje social se convierte en más individual, dirigido a sí mismo. Dicho con otras palabras, para Vygotski, a medida que el niño crece se dirige a sí mismo y utiliza para sí el habla que escucha en su medio circundante social. A los tres años la diferencia entre el lenguaje egocéntrico y el socializado es igual a cero, y a los siete años ya tenemos el lenguaje que por su estructura y funciones no se parece en absoluto al lenguaje social. Ha ocurrido la diferenciación de las dos funciones verbales: la comunicativa y la intelectual (Montealegre, 1991/1994).

En concreto, Vygotski afirma que el lenguaje egocéntrico es social por su naturaleza, ha salido de las entrañas del lenguaje social, pero aún no se ha separado de este. Se trata de una forma especial: "el lenguaje para sí". Según Vygotski, la función inicial del lenguaje es de comunicación, de vinculación social, de influencia sobre los circundantes, tanto por parte de los adultos como del niño. El lenguaje egocéntrico aparece como un estadio de tránsito del lenguaje externo (social) al interior. El lenguaje interior es la base sobre la cual se desarrolla el pensamiento lógico. Además, Vygotski considera que el lenguaje egocéntrico cumple la función intelectual de planificar y hacer inteligible la acción. El lenguaje egocéntrico se hace más frecuente y desplegado cuando ante el niño surgen dificultades que requieren un enfoque consciente y una reflexión (Montealegre, 1990, 1998).

En la investigación llevada a cabo por Montealegre (1990, 1998) sobre El papel del lenguaje en la solución de tareas espaciales por niños de edad preescolar, siguiendo los planteamientos de la psicología histórico-cultural de Vygotski, se logra el desarrollo del lenguaje para sí como planificador y regulador de la acción en niños preescolares, al facilitarles los medios simbólicos y los apoyos materiales, indispensables y accesibles para la resolución de la tarea. Al ser el lenguaje planificador una función psíquica superior, debe ser mediatizado instrumentalmente. En la experiencia espontánea del niño esto se logra gracias a la interrelación entre el lenguaje y el dibujo. Se ha observado que el niño primero dibuja y luego habla sobre lo dibujado; a una edad más avanzada habla cuando dibuja; al finalizar la edad preescolar es posible la planificación del dibujo y el acompañamiento de su realización. Así, el lenguaje, que se traslada del final al comienzo de la acción, constituye el instrumento de la planificación y regulación intelectual de la acción.

Montealegre estructura la investigación de la función planificadora del lenguaje con ayuda del método experimental genético-modelador o método del experimento formativo de Galperin (1959; 1969; 1969/1987), que reúne los métodos genético e instrumental de la psicología histórico-cultural de Vygotski.

El experimento se realizó en cuatro etapas (niños de 4 a 7 años). En todas se colocó ante el niño una maqueta en volumen de una casa, y por separado, los muebles. Durante la primera etapa -de orientación previa de la tarea - el niño se familiarizaba con cada lugar de la maqueta y tomaba conocimiento de su uso. En la segunda etapa del experimento, 
el niño trabajaba con fichas que sustituían a los muebles y con un plano previamente preparado de la casa. Se le pedía que colocara en los objetos (que se disponía a ubicar en las habitaciones) cuadrados de cartón y luego los trasladara a los correspondientes lugares del plano de la casa. Aquí, al trasladar las fichas, el niño debía decir de qué objetos se trataba y en qué habitación se disponía a ubicarlos. En la tercera etapa, el niño debía dibujar el plano de la casa y señalar con punticos los muebles en ella ubicados. Acompañaba con el lenguaje todo el proceso de realización de la acción. Finalmente, en la cuarta etapa, el niño podía tomar los muebles en sus manos y colocarlos en las habitaciones, relatando previamente al experimentador qué se disponía a hacer. Después de terminado el experimento de formación se realizó una sesión de control en la que se le presentó a los niños la tarea de organizar sobre un cuadro temático de una casa con espacios definidos (sala, comedor, cuartos, baño, etc.) diferentes figuras adhesivas de personas (papá, mamá, hijos) y de accesorios (muebles, cortinas, lámparas, etc.). La tarea se evaluaba teniendo en cuenta el número de objetos, la precisión en la colocación y la perspectiva. El resultado fundamental de la investigación fue que en todos los niños participantes del experimento se registró un lenguaje planificador. Como cualquier función psíquica superior en formación, el lenguaje planificador debe ser mediatizado instrumentalmente. El método genético-modelador usado en el experimento permitió poner de manifiesto las condiciones que llevan necesariamente a que el lenguaje adquiera la función planificadora durante la solución de tareas espaciales.

Sánchez Medina (1999), investigó en preescolares de 4 y 5 años, las acciones y el curso del habla egocéntrica en la resolución de una tarea de clasificación categorial. En la tarea se emplearon 23 tarjetas para organizar en cuatro grupos (medios de transporte, animales, alimentos y edificios) compuestos por cinco tarjetas cada uno; las tres tarjetas restantes no se podían incluir en ningún grupo. En la realización de la tarea agruparon los niños por parejas. El experimentador daba a los niños las tarjetas de modo desordenado para que las ordenaran; posteriormente, identificaba las acciones desplegadas por cada niño y analizaba si iban acompañadas o no de producciones verbales. Los enunciados de los niños fueron codificados siguiendo las siguientes dimensiones: a) plano de la emisión: hace referencia a la intención comunicativa de la emisión (social o egocéntrica); y b) perspectiva referencial: se refiere al modo en que los niños categorizan los objetos y eventos implicados en la tarea.

En esta investigación se calculó la efectividad en la resolución de la tarea, otorgando un punto por cada acción que concluyese en la colocación correcta de una tarjeta. Los resultados aportaron evidencias sobre el origen social del habla egocéntrica y mostraron su papel en la transformación de las acciones. Uno de los resultados más importantes de la investigación es el haber evidenciado el concurso del habla egocéntrica en la transformación radical de las acciones de los sujetos. Analiza, siguiendo la teoría de Vygotski, la transformación radical de las acciones de los sujetos en el paso de las acciones de un plano interpsíquico o social a uno intrapsíquico o individual. Este paso muestra la génesis progresiva de la función intelectual del signo. Esta función surge cuando el niño dirige a sí mismo signos que en etapas previas dirigía a su mundo social con intenciones comunicativas. Además, evidencia en el habla egocéntrica el uso de conceptos como instrumentos de pensamiento. Finalmente, en la investigación se concluye que el niño de edad preescolar, a medida que aprende a categorizar conceptualmente los estímulos de su entorno, y a usarlos dirigiéndolos hacia sí mismo a través del habla egocéntrica, se está dotando del poderoso instrumento de regulación de las acciones.

Controversia Piaget-Vygotski sobre el juego infantil

Shuare y Montealegre (1997) presentan en su artículo "La Situación Imaginaria, el Rol y el Simbolismo en el Juego Infantil" la Controversia Piaget-Vygotski sobre el Juego Infantil, abordado por Piaget desde el simbolismo, y por Vygotski, desde la situación imaginaria.

Piaget y el simbolismo en el juego. Piaget empezó a escribir sobre el juego en la década de los 30 y en la de los 40; se ocupa también del tema en "El lenguaje y pensamiento en el niño" (1923/1983); en "El criterio moral en el niño" (1932/1977), donde dedica el primer capítulo al desarrollo de "las reglas del juego" estudiando el juego de las canicas; en "El nacimiento de la inteligencia en el niño" (1936/1969) donde presenta una serie de observaciones sobre el juego en el período de la inteligencia sensorio-motriz. Piaget concreta su enfoque sobre el juego en su obra "La formación del símbolo en el niño" (1959/1996), en la segunda parte titulada "El juego"; y en la publicación escrita con B. Inhelder "Psicología del niño" (1969/2007) enfatiza el juego simbólico. Piaget señala que el juego simbólico es el apogeo del juego infantil.

Para Piaget, en el estadio VI del periodo sensorio-motriz comienza el juego simbólico. En este estadio VI, hay una transformación en el niño de los rituales de sus esquemas de acción sensorio-motriz en juego simbólico. El esquema simbólico de orden lúdico, desarrollado en este estadio VI, no es adaptación a lo real sino asimilación deformante (asimilación subjetiva, asimilación egocéntrica). Los primeros símbolos lúdicos aparecen cuando el niño empieza 
hablar, o sea que son contemporáneos de los primeros signos verbales. Al contrario del signo que supone una relación social, el símbolo es producto del pensamiento individual. El símbolo se basa en el parecido entre el objeto presente que juega el papel de "significante" y el objeto ausente "significado". El símbolo implica una representación de una situación no dada, la cual es evocada mentalmente. $E l$ símbolo, al presentar un parecido con su significado, es un significante motivado.

La clasificación y evolución de los juegos simbólicos, según la psicología lógica operatoria de Piaget (1959/1996) es la siguiente: Estadio I, comienza con la proyección de esquemas simbólicos y de esquemas de imitación sobre objetos nuevos. La segunda etapa del Estadio I se caracteriza por: a) asimilación simple de un objeto a otro; b) asimilación del propio cuerpo a objetos cualesquiera; se origina así un "juego de imitación". En esta segunda etapa intervienen a la vez la imitación y la asimilación simbólica. La tercera etapa, del Estadio I está constituida por: a) combinaciones simbólicas variadas: juegos manifestados en construcciones de escenas enteras (un ejemplo es el juego de muñecas); b) combinaciones compensadoras: juegos originados por miedos, celos, cólera, etc.; c) combinaciones liquidadoras: juegos consistentes en liquidar una situación desagradable; d) combinaciones simbólicas anticipatorias: juegos expresados para aceptar una orden, un consejo, etc. El fin de esta tercera etapa conduce al Estadio II, que transcurre entre los 4 y los 7 años (más o menos): el símbolo se aproxima cada vez más a lo real y comienza "el simbolismo colectivo" con adecuación de papeles o roles. Pero en esta época la socialización es bien frágil y los juegos colectivos se relacionan todavía con el simbolismo infantil.

Piaget enfatiza en las combinaciones simbólicas: a) el símbolo imaginativo, medio de expresión y de extensión, y no un fin en sí mismo; y b) el juego de imaginación, que reproduce todo lo vivido por medio de representaciones simbólicas. Piaget muestra siempre en el desarrollo cómo el sujeto va logrando dominar cada vez más los procesos de equilibración de sus estructuras cognitivas.

Vygotski y la situación imaginaria en el juego. Shuare y Montealegre (1997), respecto a la teoría de Vygotski sobre "El juego y su papel en el desarrollo psíquico del niño" (escrito en 1933, y publicado en la revista rusa "Cuestiones de Psicología", 1966), precisan el punto central de esta teoría: el juego es la realización imaginaria, ilusoria de deseos, tendencias, necesidades, impulsos, intereses, etc., que no pueden ser satisfechos inmediatamente.

Para Vygotski, la neoformación de la imaginación representa una forma humana específica de actividad de la conciencia, y como todas las funciones de esta, surge inicialmente en la acción. Vygotski señala que en la explicación del juego se deben tener en cuenta las necesidades (que entendidas en un sentido amplio, abarcan desde los impulsos hasta diversas clases de intereses) y los motivos de la actividad.

Según Vygotski, en el impulso afectivo generalizado que lleva al juego no se encuentran los gérmenes de lo simbólico, sino de la situación imaginaria (puesto que se trata de impulsos, necesidades, etc. que no pueden realizarse de manera inmediata). En la actividad de juego, el niño se libera de la atadura de la situación real; es decir, el niño comienza a actuar según motivos e impulsos que no parten de las cosas, sino que son internos.

El niño preescolar actúa en el juego según normas o reglas (mediadores instrumentales) adecuadas a la situación imaginaria; y aprende a determinar su conducta por el sentido de la situación creada (mediador semiótico).

En la edad preescolar se inicia la separación entre las cosas, el lenguaje y el pensamiento; y entre el campo visible real y el campo de sentido. Cuando el niño "cabalga" usando un palo de madera como si fuera un caballo, dicho palo es el punto de apoyo que permitirá separar el significado de la palabra caballo del animal real; en ese momento, la relación entre el objeto y el significado se invierte y este último se convierte en dominante. Pero todavía no es dominante en forma absoluta: el palo, para el preescolar, no puede ser sustituido por cualquier otro objeto, como sí lo puede hacer un adulto cuando decide que una caja de cerillas represente convencionalmente al caballo. El niño opera con los significados todavía unidos a una acción real " $\mathrm{x}$ " $\mathrm{y}$ a cierto objeto real.

Vygotski considera al juego en la edad preescolar como la actividad rectora porque en él, el niño actúa como no es capaz de actuar en la vida, se somete a las reglas implícitas de la situación imaginaria al no poder todavía someterse a las normas de la vida real, y el juego es la actividad rectora porque determina el desarrollo del niño: en el juego el niño siempre está por encima de su edad, de su conducta cotidiana habitual.

Para Vygotski, el juego es fuente de desarrollo y crea la zona de desarrollo próximo (ZDP): a) en el juego el niño siempre está por encima de su edad, de su conducta cotidiana habitual; en el juego el niño sobrepasa su edad, se adelanta a sí mismo; b) en el juego surge la acción: en un campo imaginario, en una situación ficticia, en la creación de una intención voluntaria, en la formación de un plano vital y de motivos voluntarios; y c) en el juego, la situación imaginaria es la vía que lleva al desarrollo del pensamiento abstracto, a la aceptación de las normas en ella implícita, al desarrollo de las acciones sobre cuya 
base es posible la posterior diferenciación y división entre el estudio y el juego, que se observa en la edad escolar (Shuare y Montealegre, 1997).

Vygotski, en abril de 1933, escribe a D.B. Elkonin (1978/1980) su discípulo, y le solicita idear experimentos en relación con la regla del juego agregando la imitación por estar ligada a la situación ficticia; enfatiza que todo juego de reglas es una situación ficticia.

Shuare y Montealegre (1997) exponen las contribuciones de Dannil B. Elkonin sobre el juego. A diferencia de Vygotski, para Elkonin la unidad de análisis del juego infantil, en la edad preescolar, es el papel o rol que el niño asume y las acciones ligadas a ese rol. Elkonin, en el juego de roles diferencia el tema y el contenido: el tema es aquel aspecto de la realidad que es reproducida por los niños en el juego; el contenido es lo que el niño reproduce en calidad de momento central y característico de la actividad y de las relaciones entre los adultos en su vida social real. Siendo los temas del juego sumamente diversos, el contenido es el mismo: la actividad adulta y las relaciones que surgen entre las personas. Elkonin subraya que el juego de roles es para el niño un modelador privilegiado de las relaciones que los adultos establecen entre sí en su vida social y, en este sentido, una actividad organizativa compleja.

Bodrova, Germerothg y Leong (2013) muestran la relación causal entre el juego y el desarrollo del niño, especialmente en las áreas de creatividad, razonamiento, función ejecutiva y regulación de las emociones. Partiendo de aspectos teóricos vygotskianos y post-vygotskianos plantean la diferencia entre inmadurez y madurez en el juego. Consideran que el juego de fantasía o de imaginación es el instrumento apropiado para precisar en el niño el nivel de madurez del juego y su autorregulación.

Según el nivel de madurez describen la actividad de juego realizada en el aula con los niños y el maestro. Miden: a) el uso que hacen los niños de accesorios de creación propias; b) su participación en la meta del juego; c) el rol en el juego (el papel específico); d) el uso del habla; e) la naturaleza de las interacciones de los niños en el juego. Para medir el habla-función específica, observan el número de palabras relacionadas con el tema y las frases que describen el papel propio del niño o los papeles de sus compañeros (por ejemplo: "yo seré el médico, y ustedes serán las enfermeras"); las frases relacionadas con las acciones ("estoy arreglando el coche"); las frases que describen el significado de un objeto. La evaluación del nivel de madurez del juego comprende la dimensión del docente (mediador instrumental). Al planear el juego con el docente miden: a) su gestión y su intervención dentro del juego; b) el tiempo que le asigna al juego; c) el modelado de los escenarios del juego.

En síntesis, los autores muestran la correlación entre niveles de madurez del juego y la autorregulación en el niño. Consideran que en la evaluación del juego se deben precisar: a) las características del nivel de madurez del juego, y b) los comportamientos autorregulados del niño.

Hakkarainen y Bredikyte (2008), en el estudio del juego imaginativo de roles en los preescolares, demuestra lo importante que resulta la ayuda de un adulto. En un proyecto de juego de ficción basado en cuentos populares de Finlandia observaron la acción adulta en la función imaginativa del niño. En uno de los cuentos presentados a los niños existía un punto de inflexión en un episodio donde el lobo se transforma en príncipe. La historia fue contada a los niños utilizando marionetas. El punto de inflexión fue presentado de tal manera que el títere lobo se levanta y aparece el príncipe bajo el manto. Una niña toma los títeres después de la presentación del drama, los entrega a la profesora y exige que se demuestre cómo aparece el príncipe. En el grupo, diversos cuentos fueron dramatizados. Después de la dramatización los niños esperaban qué tipo de actividad de juego les sería propuesta. Pero un cambio gradual tuvo lugar: los niños no esperaron una propuesta de juego, sino que más bien manifestaron el juego que les gustaría. Un ejemplo fue la iniciativa del "juego del barco": propusieron el tema y describieron qué papeles eran necesarios, qué cosas se requerían y cómo debía construirse el barco. El juego debía tener una trama sencilla y los niños debían jugar solos o en parejas. En estos grupos de edad preescolar existe el reto de cómo enriquecer el contenido del juego y cómo aumentar la participación del niño en una actividad conjunta.

Los autores concluyen: a) en el juego de roles, el niño actúa en situación imaginaria al escuchar los cuentos que requieren imaginar situaciones y acciones; y b) la ayuda de un adulto es importante en la investigación empírica sobre el juego de roles. En el niño de edad preescolar, el juego de roles con la ayuda del adulto lleva al desarrollo de una situación social y a cambios cualitativos debido a su intervención. Así, el juego en el preescolar define una zona de desarrollo próximo (ZDP).

Los autores, sin embargo, enfatizan que la ayuda del adulto puede no ser tan obvia cuando los niños son hábiles en papeles elementales de actuación y cuando los adultos mantienen su posición de poder en relación con los pequeños.

Al considerar importante la actividad conjunta entre los niños, los autores abordaron el juego desde el enfoque metodológico de zona de desarrollo próximo (ZDP) de Vygotski. Con el fin de estudiar la creatividad en el juego 
y la ZDP trabajaron con niños de edades entre 4-8 años la obra "El tribunal de Surmundia". El tema de la obra es el siguiente: la princesa Alexandra ha escapado y Rumpelstiltskin la persigue, los padres de la familia real están preocupados y envían a pajes y guardias a caballo en busca de la princesa. Los personajes cambian de vez en cuando, pero la línea básica de la historia sigue siendo la misma. Los roles fueron negociados y hasta discutidos, pero todo el grupo participó en la obra y nadie se quedó fuera en ningún momento.

En concreto, los autores en esta experimentación de juego partiendo del enfoque de Vygotski sobre la zona de desarrollo próximo (ZDP) desarrollaron en los niños su actividad creativa.

Controversia Piaget-Vygotski sobre el desarrollo cognitivo El nivel real de desarrollo cognitivo determina el aprendizaje, según Piaget.

Piaget e Inhelder (1969/2007) asignan cuatro factores al desarrollo mental o cognitivo: a) la maduración desempeña un papel durante todo el crecimiento mental, pero debe estar acompañada de un ejercicio funcional y de un mínimo de experiencia; b) la experiencia adquirida en la acción efectuada sobre los objetos; existe la experiencia física, que consiste en actuar sobre los objetos para abstraer sus propiedades; y la experiencia lógico-matemática, que actúa sobre los objetos para conocer el resultado de la coordinación de acciones; c) las interacciones y transmisiones sociales: la vida social determina interacciones entre el individuo que se desarrolla y el mundo que lo rodea, pero la acción social es ineficaz sin una asimilación activa del sujeto, lo cual supone instrumentos operatorios adecuados; y d) la equilibración como proceso de autorregulación o de compensaciones activas a las perturbaciones exteriores que son un obstáculo para una asimilación, para llegar a un objetivo; y como proceso de regulación retroactiva (feedback) y anticipadora. Aquí Piaget, al basarse en la cibernética, concluye que el equilibramiento por autorregulación constituye el proceso formador de las estructuras cognitivas.

En la psicología piagetiana, el nivel del desarrollo cognitivo determina el aprendizaje; es imposible acelerar tal evolución por el aprendizaje, motivo por el cual es necesario definir el nivel cognitivo antes de cualquier aprendizaje.

Piaget (1972/1980), criticando a su colaborador, el psicólogo noruego Jan Smedslund, por querer adelantar la noción de la conservación de peso mediante el aprendizaje por refuerzo externo, explica cómo los sujetos estudiados lograron un aprendizaje del resultado y se limitaron solamente a este, pero no lograron formar un instrumento de razonamiento, una lógica necesaria para la construcción del resultado. Concluye Piaget que el tiempo del desarrollo es necesario como duración y como orden de sucesión.

Inhelder, Sinclair y Bovet (1974/1975), al investigar los procesos de elaboración de determinadas nociones de conservación (cantidades numéricas, físicas y espaciales) en situaciones de aprendizaje experimentalmente controlables concluyen que: las aportaciones del medio pueden favorecer y acelerar la génesis (resultado diferente a la interpretación de maduración); los progresos son siempre función del nivel inicial del desarrollo del sujeto; los aprendizajes se insertan en los mecanismos generales del desarrollo; los obstáculos encontrados en el niño, en el curso de la elaboración de las nociones, son inherentes al pensamiento infantil; gracias a los procesos de elaboración en situaciones de aprendizaje experimentalmente controlables se puede determinar la naturaleza epistemológica de las nociones y sus relaciones estructurales; aprender es proceder a una sintesis indefinidamente renovada entre la continuidad y la novedad.

A las autoras, el aprendizaje experimental les permitió examinar los modos de interacción entre la actividad del sujeto y las aportaciones o resistencias que ofrece lo real. En la interpretación de los hechos, se adhirieron al punto de vista de Piaget sobre la delimitación de los niveles de la construcción cognoscitiva a partir de los aspectos estructurales de las nociones y operaciones subyacentes.

Piaget (1974/1975), en el Prólogo al libro de Inhelder, Sinclair y Bovet, plantea tres problemas a 'los aprendizajes como modificación del desarrollo': Primero, si las adquisiciones establecidas son estables o si, como tantos conocimientos escolares, se desvanecen al cabo de cierto tiempo; Segundo, si las aceleraciones, aparentes o reales e incluso las estables, no van acompañadas de desviaciones cuando éstas no se han obtenido mediante la utilización de los factores del desarrollo espontáneo. Tercero, si las adquisiciones obtenidas independientemente del desarrollo pueden servir como punto de partida para construcciones nuevas pero espontáneas. Es evidente, para Piaget, que no puede reducirse el desarrollo tan sólo a los aprendizajes; y que las nociones de 'niveles' y de 'competencia' se imponen como condiciones previas.

El desarrollo cognitivo potencial define el curso del aprendizaje, según Vygotski.

Vygotski (1935/1989), contrario a Piaget, considera que el nivel de desarrollo cognitivo real caracteriza el desarrollo mental retrospectivamente, mientras que el desarrollo cognitivo potencial (desarrollo próximo) caracteriza el desarrollo prospectivamente.

Vygotski $(1934 / 1979 ; 1935 / 1989)$ precisa dos niveles de desarrollo: El primero es el nivel del desarrollo real, determinado por la capacidad de resolver independientemente 
un problema sin la ayuda de los demás. Este desarrollo se mide a través de tests que establecen la edad mental.

En el nivel de desarrollo real, al no poderse establecer de modo completo el estado de desarrollo cognitivo del niño, es necesario someter a los niños a pruebas ulteriores con ayuda de preguntas-guía, ejemplos y demostraciones, y ahí empiezan las diferencias entre ellos; por ejemplo, un niño puede resolver fácilmente los tests, superando en dos años su nivel de desarrollo, mientras que otro niño llega a resolver tests que solo superan en medio año su desarrollo real.

Así, se llega al segundo nivel, al del desarrollo potencial (desarrollo próximo), determinado por la capacidad de resolver un problema bajo la guía de un adulto o con la colaboración de otro compañero más capaz, quienes actúan como instrumentos mediadores.

Vygotski establece, la Zona de Desarrollo Próximo $(Z D P)$, concebida como la distancia entre el nivel de desarrollo real y el nivel de desarrollo potencial. La zona define aquellas funciones que todavía no han madurado, pero que se hallan en proceso de maduración. Para determinar esta zona es necesaria la mediación de otra(s) persona(s). La interacción entre las personas que se encuentran en la Zona se interioriza, convirtiéndose en una nueva función del individuo. En la ZDP se da la apropiación de instrumentos culturales como el lenguaje, la lectoescritura, el cálculo, las situaciones sociales, entre otros.

La concepción de la ZDP desemboca en una nueva evaluación del papel de la imitación en el aprendizaje, no entendida la imitación en sentido mecánico, sino en relación con el pensamiento abstracto. Para Vygotski, a través de la imitación, los niños son capaces de realizar más tareas en colectividad o bajo la guía de los adultos.

Además, según Vygotski, la adquisición del lenguaje proporciona un paradigma para el problema de la relación entre el desarrollo y el aprendizaje. El esquema vygotskiano del desarrollo del lenguaje en la ontogenia es: lenguaje social - lenguaje egocéntrico - lenguaje interior. El lenguaje desde su inicio es social, es un medio de comunicación entre el niño y las personas de su entorno; posteriormente, al interiorizarse, el lenguaje social se transforma en lenguaje interior, en una función mental interna que contribuye a organizar el pensamiento del niño. El lenguaje interno y el pensamiento reflexivo surgen de las interacciones entre el niño y las personas de su entorno; dichas interacciones son fuente de desarrollo cognitivo.

Respecto al aprendizaje, Vygotski (1934/1979; 1935/1989) considera: a) el aprendizaje no equivale a desarrollo; no obstante, el aprendizaje organizado se convierte en desarrollo mental; b) existe una unidad, no una identidad, entre los procesos de desarrollo interno y los procesos de aprendizaje; y c) todo tipo de aprendizaje que el niño encuentra en la escuela tiene siempre una historia previa; d) el aprendizaje presupone una naturaleza social específica y un proceso mediante el cual los niños acceden a la vida intelectual de aquellos que les rodean.

Para Vygotski, el aprendizaje del niño comienza mucho antes del aprendizaje escolar. Todo el aprendizaje del niño en la escuela tiene una prehistoria. El autor presenta los siguientes ejemplos: a) el lenguaje escrito: antes de comenzar la educación formal existe una prehistoria del lenguaje escrito, manifestándose en la aparición de una serie de instrumentos psicológicos: los gestos, escritura en el aire; los garabatos, en los que el niño no está dibujando el objeto en sí, sino que está fijando en el papel los gestos con los que él mismo representa a dicho objeto; y la representación de objetos en el juego simbólico; y b) la aritmética: antes del aprendizaje de la aritmética en la escuela, el niño ya tiene una pre-escuela de la aritmética, ha adquirido cierta experiencia referente a la cantidad y se ha relacionado ya con operaciones de división y adición complejas y sencillas.

Lógicamente, este proceso de aprendizaje que se produce antes de entrar el niño a la escuela difiere de manera esencial de los procesos que se adquieren durante la enseñanza escolar. El aprendizaje escolar aporta algo completamente nuevo al curso del desarrollo. Además, siguiendo la psicología histórico-cultural, el aprendizaje debe planearse sobre la base del desarrollo cognitivo potencial y la utilización de instrumentos mediadores.

Montealegre $(2011,2013)$, partiendo de la psicología histórico-cultural en lo relacionado al desarrollo cognitivo, llevó a cabo un procedimiento y una situación experimental en estudiantes de psicología de $1^{\circ}, 4^{\circ}, 5^{\circ}$ semestre con el objetivo central de desarrollar la Función Psicológica Superior (FPS) de Solución de Problemas Cognitivos (SPC) mediante la realización de tareas cognitivas sobre: pensamiento productivo (creativo); juegos y entretenimientos matemáticos; y conflicto sociocognitivo, originado en el análisis de hechos y situaciones sociales.

Otros objetivos de la investigación fueron: a) formar acciones mentales mediadas por instrumentos: materiales, semióticos (el lenguaje) y otros seres humanos portadores de significados en interacción social (zona de desarrollo próximo); y b) aplicar técnicas instrumentales, estrategias o procedimientos heurísticos (procedimientos falibles pero confiables) y situaciones de interacción social en la ejecución de las tareas cognitivas.

La parte experimental de la investigación consistió en seis sesiones de intervención de tres horas seguidas cada 
una, en las cuales se realizaban las tareas cognitivas mencionadas: a) bajo la guía de una persona (un profesor o un par más capaz) que orientaba el trabajo; b) en grupos pequeños (15 personas máximo) que permitían ser orientados en la realización de las tareas cognitivas y dividirse en grupos de tres personas. En total hubo 18 horas de intervención.

El método del experimento formativo de la psicología histórico-cultural se utilizó para el desarrollo cognitivo en las Sesiones de Intervención sobre Pensamiento Productivo; y sobre Juegos y Entretenimientos Matemáticos. El método se apoya en la teoría (modelo) de la formación planificada de las acciones mentales (Galperin,1959;1969;1969/1987), y comprende los siguientes pasos: a) Base Orientadora de la Acción: se solicita a los sujetos una diagramación de las condiciones del problema y una diagramación del estado inicial y del estado final del problema; b) Tareas con Apoyo en Objetos Materiales: se induce a los sujetos a diagramar los estados intermedios en la ejecución de la tarea y a seguir una serie de instrucciones y pasos para poder llegar a la solución final de la tarea; c) Presentación de los Ejercicios en Grupo: los sujetos explican sus respuestas teniendo en cuenta los instrumentos mediadores.

Además, se realizan unas sesiones de intervención sobre conflicto sociocognitivo con base en las conceptualizaciones acerca del conflicto estructurante o creativo de una actividad cognitiva de la Psicología Social Cognitiva, derivada de la psicología de Piaget; y de la Zona de Desarrollo Próximo $(Z D P)$, de la psicología histórico-cultural de Vygotski. Se induce a los estudiantes a dominar conceptos y hechos sociales relacionados con situaciones de discriminación social, de género y de sexo. Los sujetos deben construir procesos de solución y análisis de manera consensuada.

En la investigación se concluye que existe la necesidad de desarrollar, en estudiantes de psicología y en universitarios en general, procesos cognitivos a través de los cuales se pueda lograr mayor asimilación y aprendizaje de tareas cognitivas indispensables en la actividad académica (Montealegre, 2011, 2013).

Hedegaard y Chaiklin (2005), partiendo de la tesis vygotskiana sobre "el aprendizaje organizado es fuente de desarrollo mental", llevaron a cabo un programa de enseñanza aprendizaje local-radical (local-relevante culturalmente), como actividad extracurricular, en un grupo de niños puertorriqueños de educación primaria del Este de Harlem, de la ciudad de Nueva York. Lo local-relevante culturalmente se refiere a explorar las condiciones históricas y culturales de la comunidad de origen y de la comunidad donde viven los niños. Partiendo de la psicología históricocultural de Vygotski, consideran: a) la interacción entre desarrollo mental y aprendizaje (los procesos de aprendizaje proporcionan la base para el desarrollo de procesos psíquicos); b) la enseñanza experimental de los conocimientos de las materias desde una perspectiva teórico-práctica que apunte al aprendizaje de los conceptos de manera teóricodialéctico sobre la base del desarrollo histórico-cultural de la humanidad (general o local); c) la interacción entre los conceptos cotidianos (conceptos dados en la vida diaria) y los conceptos científicos (aprendidos en la instrucción formalizada). La enseñanza estuvo encaminada a desarrollar la habilidad de relacionar los conceptos cotidianos con los conceptos científicos; y a utilizar el contenido de las materias aprendidas en el análisis de actividades locales de la comunidad. El objetivo de este trabajo de enseñanza-aprendizaje local radical es desarrollar en los niños una comprensión teórico-dialéctica de conocimientos sobre ciencias sociales (historia y estudios sociales) a partir de temas centrales de la vida cotidiana de los mismos niños y de la comunidad local (barrio, vecinos). La meta es mostrar cómo se puede conducir la enseñanza de una materia que simultáneamente se desprenda de la situación histórica del participante, y a la vez contribuya a su propio desarrollo mental. La enseñanza local-radical (instrumento mediatizador) se realizó en 37 sesiones y comenzó a partir de una serie de preguntas sobre los orígenes de la comunidad local: ¿Cuáles son nuestras raíces?, ¿Cuáles son las características de la sociedad en la cual vivimos hoy?, ¿Cómo nos relacionamos con esta sociedad como miembros de la comunidad de Puerto Rico? Posteriormente realizaron una serie de sesiones en donde los niños debían investigar la vida familiar y las condiciones de vida a comienzos del siglo XX en Puerto Rico y en Nueva York. Luego, debían investigar sobre la historia de su ciudad actual, Nueva York, para pasar a la investigación sobre su propia comunidad. Cada sesión fue grabada por un observador participante, quien también escribía notas de campo sobre lo que sucedía. Se observó, en este experimento de enseñanza, cómo los niños empezaron a ser capaces de trabajar con todas las relaciones y conceptos del modelo central (comunidad, condiciones de vida, familia y recursos) y empezaron a comprender problemas concretos relativos a su comunidad local (barrio, vecinos). En la investigación se enfatiza en la necesidad de formular los tres aspectos o perspectivas de una práctica institucional educativa: 1 . Lo social, refleja el desarrollo histórico de tradiciones e intereses en una determinada sociedad que se formalizan por medio de leyes y regulaciones: 2. Lo general, orienta lo teorético (nivel de abstracción y generalización) de las actividades institucionales; 3 . Lo individual, caracteriza las actividades compartidas de los individuos en instituciones específicas. Cada aspecto-social, general, e individual- es condición para los otros. Consideran que los tres aspectos 
están presentes en cualquier práctica cultural concreta. Los investigadores, al precisar los datos obtenidos, llegaron a la conclusión de que el desarrollo de los niños comprende tres formas generales de conocimiento: empírico-paradigmático, narrativo-dialéctico y teórico-dialéctico

\section{DISCUSIÓN}

En este trabajo se presenta una serie de reflexiones teóricas e investigativas sobre la psicología lógica operatoria de Piaget y la psicología histórico-cultural de Vygotski, respecto al lenguaje egocéntrico, al juego infantil y al desarrollo cognitivo. Se orienta el debate a partir de "la unidad de análisis": la lógica operatoria y la equilibración, en Piaget; y la acción mediatizada por instrumentos, en Vygotski.

La discusión entre estas dos psicologías permite precisar el curso de la acción en el desarrollo del ser humano: 1 . En la psicología piagetiana, la acción de la lógica operatoria en la interpretación de los procesos psíquicos; y la explicación del factor de equilibración en el desarrollo, basado en las compensaciones activas realizadas por el sujeto como reacción a las modificaciones exteriores. En la psicología piagetiana, la acción se desarrolla de lo individual a lo social; lo social se desarrolla a partir de la coordinación de acciones individuales. 2. En la psicología vygotskiana, la acción de lo histórico-cultural en el desarrollo de las funciones psíquicas superiores (FPS). En el conjunto de la teoría vygotskiana, el eje como espiral dialéctico que organiza todos los demás conceptos es el historicismo. Shuare y Shuránova (1996) analizan en la obra vygotskiana tres conceptualizaciones a partir del historicismo: el tiempo humano es historia, tanto en lo individual como en lo social; el desarrollo histórico de los fenómenos psíquicos mantiene una relación de dependencia con la actividad social; y las funciones psíquicas superiores son el producto de la compleja interacción del individuo con el mundo, interacción mediatizada por los objetos creados por el ser humano, En la psicología vygotskiana, la acción se desarrolla de lo social a lo individual; lo social está desde el principio en la interacción del niño con el adulto y se desarrolla hacia el manejo de las acciones individuales.

Además, las controversias entre la psicología lógica operatoria de Piaget y la psicología histórico-cultural de Vygotski permiten precisar el desarrollo mental o cognitivo: 1. La psicología lógica operatoria de Piaget caracteriza el desarrollo cognitivo retrospectivamente, delimita los niveles cognitivos a partir de los aspectos estructurales de las nociones y operaciones subyacentes. El tiempo del desarrollo es necesario como duración y como orden de sucesión. Determina los estadios del desarrollo mental desde el proceso de equilibración de sus estructuras cognitivas. 2. La psicología histórico-cultural de Vygotski caracteriza el desarrollo cognitivo prospectivamente; al respecto define una zona de desarrollo próximo (ZDP) que establece la distancia entre el nivel de desarrollo real y el nivel de desarrollo potencial.

Los aportes de este trabajo "Controversias PiagetVygotski en Psicología del Desarrollo" son: 1. Partir de la concepción de "unidad de análisis" en cada una de estas teorías y determinar sus aspectos teóricos y metodológicos. 2. Precisar una reflexión mostrando aportes de investigaciones neo-vygotskinas al lenguaje egocéntrico, al juego infantil y al desarrollo cognitivo, en donde la investigación va dirigida a desarrollar funciones psicológicas superiores $\mathrm{y}$ acciones individuales.

En síntesis, entre estas dos psicologías del desarrollo, la base de toda la discusión es su concepción epistemológica: 1 . En la teoría piagetiana, el "estructuralismo lógico constructivista": construcción progresiva de estructuras operatorias que se equilibran unas detrás de otras, apoyándose siempre en las siguientes, llenando lagunas y reequilibrándose en un terreno más amplio. El estudio psicogenético piagetiano enfatiza en el desarrollo de las estructuras operatorias de la inteligencia durante los primeros quince años de la existencia del sujeto; 2. En la teoría vygotskiana, la "dialéctica materialista e histórica", la cual define la esencia de la psiquis humana por el desarrollo de la sociedad, por el desarrollo individual y por su carácter mediatizado. La acción mediada por instrumentos (mediación instrumental) lleva al análisis sociocultural y al desarrollo de procesos cognitivos en el ser humano. Las transformaciones que experimenta la psiquis humana son tanto estructurales como funcionales.

\section{REFERENCIAS}

Bodrova, E., Germeroth, C., y Leong, D.J. (2013). Play and self-regulation: Lessons from Vygotsky. American Journal of Play, 6(1), 111-123.

Elkonin, D.B. (1980). Anexo: Fragmento de los apuntes de L.S. Vygotski para unas conferencias de psicología de los párvulos. En D. B. Elkonin. Psicología del juego (págs. 269-276; V. Uribes, Trad.). Madrid: Pablo del Río, Editor. (Trabajo original publicado en 1978).

Flavell, J.H., Botkin, P.T., Fry, C.L., Wright, J.C., y Jarris. P.E. (1968). The development of role-taking and communication skills in children. New York: Wiley.

Galperin, P.Y. (1959). Desarrollo de las investigaciones sobre la formación de las acciones mentales. Revista La Cien- 
cia Psicológica en la URSS (Psijologuicheskaia Naúka v SSSR), 1, 446-463.

Galperin, P.Y. (1969). Stages in the development of mental acts. En M. Cole, y I. Maltzman (Eds.). A handbook of contemporary Soviet Psychology. New York: Basic Books.

Galperin, P.Y. (1987). Sobre la investigación del desarrollo intelectual del niño. En M. Shuare, y V. Davidov (Eds.), La psicología evolutiva y pedagógica en la URSS (págs. 125142; M. Shuare, Trad.). Moscú: Progreso. (Trabajo original publicado en 1969).

Hakkarainen, P., y Bredikyte, M. (2008). The zone of proximal development in play and learning. Cultural-Historical Psychology, 4. Recuperado el 11 de junio 2014, de http:// lchc.ucsd.edu/MCA/Mail/xmcamail.2010_11.dir/pdf1GzfHp0kIH.pdf.

Hedegaard, M. y Chaiklin, S. (2005). Radical-local teaching and learning. A cultural-historical approach. Aarhus, Dinamarca: Aarhus University Press.

Inhelder, B., Sinclair, H., y Bovet, M. (1975). Aprendizaje y estructuras del conocimiento (L. E. Echevarria Rivera, Trad.). Madrid: Ediciones Morata, S.A. (Trabajo original publicado en 1974).

Kohlberg, L., Yaeger, J., y Hjertholm, E. (1968). Private speech: Four studies and review of the theories. Child Development, 39, 691-736.

Montealegre, R. (1990). Papel del lenguaje en la solución de tareas espaciales por niños de edad preescolar. Revista Latinoamericana de Psicología, 22(2), 239-252.

Montealegre, R. (1994). El papel del lenguaje y del pensamiento en el niño de edad preescolar según Piaget y Vygotski. En R. Montealegre, Vygotski y la concepción del lenguaje (págs. 72-82). Bogotá: Serie Cuadernos de Trabajo, Facultad de Ciencias Humanas, Universidad Nacional de Colombia. (Trabajo original publicado en 1991).

Montealegre, R. (1998). El experimento formativo en el estudio del lenguaje egocéntrico. Revista Latinoamericana de Psicología, 30(2), 261-277.

Montealegre, R. (2011). La solución de problemas cognitivos en estudiantes de psicología. Revista Acta Colombiana de Psicología, 14(1), 119-138.

Montealegre, R. (2013). Manual orientador en solución de problemas cognitivos. Bogotá: Serie II-Nº6, Centro de Investigaciones sobre Dinámica Social, Universidad Externado de Colombia.

Piaget, J, (1967). Programas y métodos de la epistemología genética. En J. Piaget, Psicología, lógica y comunicación (págs. 21-118; N. Bastard, Trad.) Buenos Aires: Ediciones Nueva Visión: Colección Ensayos. (Trabajo realizado en el Centro Internacional de Epistemología Genética durante el año 1955-56).

Piaget, J. (1969). La naissance de l'intelligence chez l'enfant. Neuchatel-Paris: Delachaux \& Niestlé. (Trabajo original publicado en 1936).
Piaget, J. (1973). El juicio y el razonamiento en el niño. Estudio sobre la lógica del niño (II) (M. Riani, Trad.). Buenos Aires: Guadalupe. (Trabajo original publicado en 1924).

Piaget, J. (1975) Prólogo. En B. Inhelder, H. Sinclair, y M. Bovet. Aprendizaje y estructuras del conocimiento (págs. 13-17; L. E. Echevarria Rivera, Trad). Madrid: Ediciones Morata, S.A. (Trabajo original publicado en 1974).

Piaget, J. (1977). El criterio moral en el niño (N. Vidal, Trad.). Barcelona: Fontanella. (Trabajo original publicado en 1932).

Piaget, J. (1980). Problemas de Psicología Genética (M.A. Quintanilla). Barcelona: Ariel. (Trabajo original publicado en 1972).

Piaget, J. (1981). Seis estudios de psicología (N. Petit, Trad.). Barcelona: Seix Barral, S.A. (Trabajo original publicado en 1964).

Piaget, J. (1983). El lenguaje y el pensamiento en el niño. Estudio sobre la lógica del niño (I) (M. Riani, Trad.). Buenos Aires: Guadalupe. (Trabajo original publicado en 1923).

Piaget, J. (1985). Commentaires sur les remarques critique de Vygotski. En B. Schneuwly, y J.P. Bronckart (Eds.), Vygotsky aujourd'hui (Cap. 6, págs.120-138). Neuchatel-Paris: Delachaux \& Niestlé. (Trabajo original publicado en 1959).

Piaget, J. (1996). La formación del símbolo en el niño. Segunda Parte: El Juego (págs.121-292) - (J. Gutiérrez, Trad.). México: Fondo de Cultura Económica. (Trabajo original publicado en 1959).

Piaget, J., e Inhelder, B. (2007). Psicología del niño (L.H. Alfonso, Trad.). Madrid: Morata. (Trabajo original publicado en 1969).

Piaget, J. (2012). La equilibración de las estructuras cognitivas. Problema central del desarrollo (E. Bustos, Trad.). España: Siglo Veintiuno Editores. (Trabajo original publicado en 1975).

Sánchez Medina, J. (1999). Pensamiento y Lenguaje. Habla egocéntrica y regulación de las acciones. Madrid - Buenos Aires: Miño y Dávila Editores.

Shuare, M. O., y Montealegre, R. (1997). La situación imaginaria, el rol y el simbolismo en el juego infantil. Revista Colombiana de Psicología, $N^{\circ}$ 5-6, 82-88.

Shuare, M.O., y Shuránova, I. Yu. (1996). Lev S. Vygotski: Nuevos Desarrollos. En R. Montealegre (Ed.), El Siglo de Vygotski y de Piaget (págs. 561-568). Bogotá: Fundación para el Avance de la Psicología.

Vygotski, L.S. (1966). El juego y su papel en el desarrollo psíquico del niño. Revista Cuestiones de Psicología 6, 62-75 (Voprosy Psijologui 6, 62-75), (Trabajo original escrito en 1933).

Vygotski, L.S. (1979). Aprendizaje y desarrollo intelectual en la edad escolar (Ma. E. Benítez, Trad. de la versión italiana). En Luria, Leóntiev, Vygotski, Psicología y Pedagogía (págs. 23-39). Madrid: AKAL Editor. (Trabajo original escrito en 1934, publicado en 1956). 
Vygotski, L.S. (1983). Historia del desarrollo de las funciones psíquicas superiores. En L.S. Vygotski, Obras Escogidas (Sobranie Socheniy), Tomo Tercero (Tom Tretiy), Moscú: Pedagógica (Moskva: Pedagóguika). (Trabajo original escrito en 1931, publicado en 1960).

Vygotski, L.S. (1984). Instrumento y Signo en el desarrollo del niño. En L.S. Vygotski, Obras Escogidas (Sobranie Socheniy), Tomo Sexto (Tom Shestoy). Moscú: Pedagógica (Moskva: Pedagóguika). (Trabajo original publicado en 1930).

Vygotski, L.S. (1989). El desarrollo de los procesos psicológicos superiores (págs. 123-140; y 159-178; S. Furió, Trad. de la versión en inglés "Mind in Society. The Development of Higher Psychological Processes"). Barcelona: CríticaGrijalbo (Trabajo publicado póstumamente en 1935).
Vygotski, L.S. (1991). El método instrumental en psicología (En: Problemas Teóricos y Metodológicos de la Psicología). En L.S. Vygotski, Obras Escogidas I. (págs. 65-70; J.M. Bravo, Trad.). Madrid: Aprendizaje Visor. (Conferencia original 1930, publicada en 1960).

Vygotski, L.S. (1993). Pensamiento y Lenguaje. En L.S. Vygotski, Obras Escogidas II. (págs. 11-348; J.M. Bravo, Trad.). Madrid: Aprendizaje Visor. (Trabajo original publicado en 1934).

Wertsch, J.V. (1988). Vygotsky y la formación social de la mente (J. Zanón y M. Cortés, Trads.) Barcelona: Paidós. (Trabajo original publicado en 1985).

Zínchenko, V.P. (1993). La teoría psicológica histórico-cultural: experiencia de amplificación. Revista Cuestiones de Psicología (Voprosy Psijologuii), 4, 5-19. 